\title{
TRANSLATION-INVARIANT DIRICHLET ALGEBRAS ON COMPACT GROUPS
}

\author{
DANIEL RIDER ${ }^{1}$
}

1. Introduction. Let $G$ be a compact group and $C(G)$ the space of continuous functions on $G$. This paper is concerned with characterizing all translation-invariant,$^{2}$ uniformly closed Dirichlet ${ }^{3}$ subalgebras of $C(G)$. If $G$ is Abelian and connected then such an algebra exists with the additional property that it contains no real functions other than the real constants. In fact, for each ordering of the dual group of $G$, the space of functions analytic with respect to this order is such an algebra. As a converse we have

THEOREM 1. If $C(G)$ contains a translation-invariant, uniformly closed Dirichlet algebra which contains no nonconstant real functions, then $G$ is Abelian and connected.

It is then possible to obtain the following characterization.

THEOREM 2. Let $A$ be a translation-invariant, uniformly closed Dirichlet subalgebra of $C(G)$. There is a closed, connected subgroup, $H$, of the center of $G$ and an order, $P$, on the dual group of $H$ such that $A$ consists of all continuous functions which are analytic (with respect to $P$ ) on the cosets of $H$.

Conversely, to every such subgroup and order corresponds such an algebra.

$H$ turns out to be the maximal antisymmetric set of $A$ which contains the identity element of $G$. The theorems are obtained by considering the relations between translation-invariant algebras and the "hypergroup" structure associated with $G$.

2. Hypergroups. For a compact group $G$, let $\hat{G}$ denote the set of equivalence classes of irreducible unitary representations of $G$. For $\alpha \in \hat{G}, T_{\alpha}$ is a member of the class $\alpha, \chi_{\alpha}$ is the character of the class, and $d(\alpha)$ the degree. $\hat{G}$ has a hypergroup structure (cf. Helgason [3]) in the following sense. If $\alpha, \beta \in \hat{G}$ then $T_{\alpha} \otimes T_{\beta}$ has a decomposition

Received by the editors April 6, 1966.

1 This research was supported in part by Air Force Office of Scientific Research Grant A-AFOSR 335-63.

${ }^{2}$ Closed under both right and left translations.

${ }^{3}$ The real parts of the functions in the subalgebras are uniformly dense in the real continuous functions and the subalgebra contains 1 . 
into irreducible unitary components. If $\mu_{\alpha, \beta}(\gamma)$ is the number of times $T_{\gamma}$ appears in this decomposition then

$$
\chi_{\alpha}(g) \chi_{\beta}(g)=\sum_{\gamma \in \hat{G}} \mu_{\alpha, \beta}(\gamma) \chi_{\gamma}(g) \quad(g \in G) .
$$

A subset $\mathfrak{H C}$ of $\hat{G}$ is called a subhypergroup if $\alpha, \beta \in \mathfrak{F C}$ and $\mu_{\alpha, \beta}(\gamma)$ $\neq 0$ imply $\gamma \in \mathcal{H}$. A subhypergroup is normal if $\alpha \in \mathcal{H}$ implies $\bar{\alpha} \in \mathcal{H}$ where $T_{\bar{\alpha}}$ is the representation conjugate to $T_{\alpha}$. If $I$ is the unit representation of $G$ then $\mu_{\alpha, \bar{\alpha}}(I)=1$ so that $I$ belongs to every normal subhypergroup. Also $\chi_{\bar{\alpha}}=\bar{\chi}_{\alpha}$.

If $G$ is Abelian, then $\hat{G}$ is a group and its subhypergroups are just its subsemigroups. The normal subhypergroups of $\hat{G}$ are the subgroups of $\hat{G}$.

The following lemma corresponds to the decomposition of a subsemigroup of an Abelian group into a subgroup and a semigroup which does not contain the identity.

Lemma 3. Let $\operatorname{He}$ be a subhypergroup of $\hat{G}$,

$$
\mathfrak{H}_{1}=\{\alpha: \alpha \in \mathfrak{H}, \bar{\alpha} \in \mathfrak{H}\} \text { and } \mathfrak{H}_{2}=\mathfrak{H C}_{\mathfrak{H}} \text {. }
$$

(a) $\mathfrak{H C}_{1}$ and $\mathfrak{H C}_{2}$ are subhypergroups.

(b) $\mathfrak{H}_{1}$ is normal.

(c) If $\alpha \in \mathcal{H C}, \beta \in \mathcal{H C}_{2}$ and $\mu_{\alpha, \beta}(\gamma) \neq 0$ then $\gamma \in \mathcal{H C}_{2}$.

Clearly $\mathfrak{H}_{1}$ is nonempty if and only if $I \in \mathfrak{H}$.

Proof. Since $\mu_{\alpha, \beta}(\gamma)=\int_{G} \chi_{\alpha}(g) \chi_{\beta}(g) \chi_{\bar{\gamma}}(g) d g$ (dg is Haar measure on $G$ ) it follows that

(1) $\mu_{\alpha, \beta}(\gamma)=\mu_{\alpha, \bar{\gamma}}(\bar{\beta})$ and

(2) $\mu_{\alpha, \beta}(\gamma)=u_{\alpha, \bar{\beta}}(\bar{\gamma})$.

If $\alpha \in \mathcal{H C}, \beta \in \mathcal{F C}_{2}$ and $\mu_{\alpha, \beta}(\gamma) \neq 0$ then $\gamma \in \mathcal{H C}$. Now if $\bar{\gamma} \in \mathcal{F C}$ then it follows from (1) that $\bar{\beta} \in \mathcal{H C}$ contrary to assumption. This proves (c).

It follows from (c) that $\mathfrak{H C}_{2}$ is a subhypergroup. On the other hand, if $\alpha, \beta \in \mathcal{H}_{1}$ and $\mu_{\alpha, \beta}(\gamma) \neq 0$ then it follows from (2) that $\gamma, \bar{\gamma} \in \mathcal{H}$. Thus $\gamma \in \mathfrak{H}_{1}$ so that $\mathfrak{H}_{1}$ is a subhypergroup. It is normal by its definition.

If $M$ is a subset of $G$ then $M^{\perp}$ denotes the set of $\alpha \in \hat{G}$ such that $T_{\alpha}(g)=E$, the identity transformation, for all $g \in M$. If $\mathfrak{H C} \subset \hat{G}, \mathfrak{F}^{\perp}$ is the set of $g \in G$ such that $T_{\alpha}(g)=E$ for all $\alpha \in \mathcal{H}$. Duality theorems concerning $M^{\perp}$ and $\mathfrak{H}^{\perp}$ are discussed in [3].

A translation-invariant, uniformly closed subalgebra of $C(G)$ will be called an $I$-algebra on $G$. A translation-invariant, uniformly closed linear subspace of $C(G)$ is completely determined by the irreducible 
characters it contains. If $A$ is an $I$-algebra these characters form a subhypergroup. If $A$ is an $I$-algebra, let $A^{\prime}=\left\{\alpha: \chi_{\alpha} \in A\right\}$. If $\mathcal{T C}$ is a subhypergroup, let $\mathcal{F C}^{\prime}$ be the translation-invariant, uniformly closed linear subspace generated by $\left\{\chi_{\alpha}: \alpha \in \mathfrak{H}\right\}$. The following is easily seen.

Lemma 4. (a) $A^{\prime}$ is a subhypergroup and $\mathfrak{H}^{\prime}$ is an I-algebra.

(b) $\left(A^{\prime}\right)^{\prime}=A,\left(\mathfrak{H C}^{\prime}\right)^{\prime}=\mathfrak{H C}$.

(c) $A$ is a Dirichlet algebra if and only if

$$
A^{\prime} \cup\left(\bar{A}^{\prime}\right)=\hat{G},\left(\bar{A}^{\prime}=\left\{\bar{\alpha}: \alpha \in A^{\prime}\right\}\right) .
$$

(d) $A$ contains no nonconstant real functions if and only if $A^{\prime} \cap \bar{A}^{\prime}$ $\subset\{I\}$.

3. Antisymmetric sets. Let $A$ be a uniformly closed subalgebra of $C(G)$ which contains the constants. A subset $K$ of $G$ is called an antisymmetric set of $A$ if $f \in A$ and $f$ real valued on $K$ imply $f$ is constant on $K$ (cf. Bishop [1], Glicksberg [2]). It is shown in [1] that maximal antisymmetric sets exist and form a disjoint cover of $G$. If $A$ is also translation-invariant, it is easily seen that the maximal antisymmetric set of $A$ which contains the identity element of $G$ is a normal subgroup of $G$ and that the other maximal antisymmetric sets are the cosets of this group.

Lemma 5. Let $A$ be an I-algebra on $G$ which contains the constants. If $A_{1}^{\prime}$ is the unique maximal normal subhypergroup contained in $A^{\prime}$ (cf. Lemma 3 ), then $\left(A_{1}^{\prime}\right)^{\perp}$ and its cosets are the maximal antisymmetric sets of $A$.

$\left(A_{1}^{\prime}\right)^{\perp}$ is a normal subgroup by [3].

Proof. Let $K$ be the closed normal subgroup of $G$ which along with its cosets forms the maximal antisymmetric sets of $A$. Suppose $\alpha \in K^{\perp}$, then $\chi_{\alpha}$ and $\chi_{\bar{\alpha}}$ are constant on the cosets of $K$ so that, by Bishop's theorem [1], $\chi_{\alpha}$ and $\chi_{\bar{\alpha}}$ belong to $A$. Thus $\alpha \in A_{1}^{\prime}$. Conversely, if $\alpha \in A_{1}^{\prime}$ then $\chi_{\alpha}, \chi_{\bar{\alpha}} \in A$. Now $\chi_{\alpha}+\chi_{\bar{\alpha}}$ is real on $G$ (hence on $K$ ) so that $\chi_{\alpha}+\chi_{\bar{\alpha}}$ is constant on $K$. But this implies $\chi_{\alpha}(g)=\chi_{\alpha}(e)$ $=d(\alpha)(g \in K)$ so that $T_{\alpha}(g)=E(g \in K)$ and $\alpha \in K^{\perp}$. Thus $K^{\perp}=A_{\mathbf{1}}^{\prime}$ so that, by Helgason's duality theorem [3], $K=\left(K^{\perp}\right)^{\perp}=\left(A_{1}^{\prime}\right)^{\perp}$.

REMARK 6. Let $H=\left(A_{1}^{\prime}\right)^{\perp}$. Now by [2] $A \mid H$, the functions in $A$ restricted to $H$, is uniformly closed in $C(H)$. Suppose $A$ is also a Dirichlet algebra, then $A \mid H$ is an $I$-Dirichlet algebra on $H$ and since $H$ is an antisymmetric set of $A, A \mid H$ contains no nonconstant real 
functions. The following section will show that in this case $H$ must be Abelian and connected.

4. Proof of Theorem 1 . We will say that $\hat{G}$ can be ordered if there is a subhypergroup $\mathfrak{F C} \subset \hat{G}$ such that

$$
\mathfrak{H C} \overline{\mathfrak{H C}}=\{I\} \text { and } \mathfrak{H} \cup \overline{\mathfrak{H C}}=\hat{G} \text {. }
$$

If $G$ is Abelian, so that $\hat{G}$ is a group, this agrees with the usual definition and $\hat{G}$ can be ordered if and only if $G$ is connected [4, p. 197].

$\hat{G}$ can be ordered precisely when there is an analytic structure on $G$ in the following sense.

LEMмA 7. The following are equivalent:

(a) $\hat{G}$ can be ordered.

(b) There is an I-Dirichlet subalgebra of $C(G)$ which contains no nonconstant real functions.

Proof. If $\mathfrak{F}$ orders $\hat{G}$ then $\mathfrak{F}^{\prime}$ satisfies (b). Conversely, if $A$ satisfies (b) then $A^{\prime}$ orders $\hat{G}$.

If for some $\alpha \in G, \alpha \neq I$, and positive integer $n$ we have $\int \chi_{\alpha}^{n} \neq 0$, then every subhypergroup containing $\alpha$ contains $\bar{\alpha}$. This follows since $\int \chi_{\alpha}^{n}$ is the number of times $T_{\bar{\alpha}}$ appears in the tensor product of $T_{\alpha}$ with itself $n-1$ times. In such a case $\hat{G}$ certainly cannot be ordered.

Lemma 8. If $G$ is non-Abelian, there is $\alpha \in \hat{G}, \alpha \neq I$, and positive integer $n$ such that $\int \chi_{\alpha}^{n} \neq 0$.

Theorem 1 follows immediately from Lemmas 7 and 8 .

Proof of Lemma 8. Since $G$ is non-Abelian there is $\beta \in \hat{G}$ with $d(\beta)>1$. Let $I, T_{\alpha_{1}}, \cdots, T_{\alpha_{m}}$ be the irreducible components of $T_{\beta} \otimes T_{\bar{\beta}}$. Since $T_{\beta}$ is irreducible, $I$ appears exactly once. For $g \in G$, $T_{\beta} \otimes T_{\bar{\beta}}(g)$ has one as an eigenvalue at least $d(\beta)$ times. Hence, since $d(\beta)>1$, at least one of the $T_{\alpha_{i}}(g)$ has one as an eigenvalue. Thus, for some $i,\left\{g: T_{\alpha_{i}}(g)\right.$ has one as an eigenvalue $\}$ has positive measure. If $F(g)$ is the number of times one is an eigenvalue of $T_{\alpha_{i}}(g)$ then $\int F>0$.

It is easily seen that

$$
F(g)=\lim _{N \rightarrow \infty} \frac{1}{N} \sum_{n=1}^{N} \chi_{\alpha_{i}}\left(g^{n}\right) \quad(g \in G) .
$$

Now since $(1 / N) \sum_{n=1}^{N} \chi_{\alpha_{i}}\left(g^{n}\right)$ is a uniformly bounded sequence of functions converging to $F$ we have that $(1 / N) \sum_{n=1}^{N} \int \chi_{\alpha_{i}}\left(g^{n}\right) d g$ $\rightarrow \int F$. Hence for some positive integer $n, \int \chi_{\alpha_{i}}\left(g^{n}\right) d g \neq 0$. Now the $I$-algebra generated by $\chi_{\alpha_{i}}$ contains $f(g)=\chi_{\alpha_{i}}\left(g^{n}\right)$. Thus since $\int f \neq 0$, 
this $I$-algebra contains the constants. This is equivalent to saying the hypergroup generated by $\chi_{\alpha_{i}}$ contains I which is equivalent to saying $\int \chi_{\alpha_{i}}^{n} \neq 0$ for some positive integer $n$.

5. Proof of Theorem 2. From Remark 6 and Theorem 1 it follows that if $A$ is an $I$-Dirichlet algebra on $G$ and $H$ is the maximal antisymmetric set of $A$ containing the identity then $H$ is a closed, connected, normal Abelian subgroup of $G$. Every irreducible representation of $H$ is a component of some irreducible representation of $G$ restricted to $H$. Let $P=\left\{\phi \in \hat{H}: \phi\right.$ is a component of $\left.\alpha \mid H, \alpha \in A^{\prime}\right\}$. $H$ is Abelian so that the irreducible representations and characters of $H$ are the same. 1 will denote the identity of $\hat{H}$. Since $A^{\prime}$ is a subhypergroup, $P$ is a semigroup. Also since $A$ is a Dirichlet algebra, Lemma 4(c) implies $P \cup \bar{P}=\hat{H}$.

Suppose $\phi \in P$ and $\bar{\phi} \in P$. Then there are $\alpha, \beta \in A^{\prime}$ with $\phi, \bar{\phi}$ components of $\alpha \mid H$ and $\beta \mid H$ respectively. Thus for some $\gamma$, with $\mu_{\alpha, \beta}(\gamma) \neq 0,1$ is a component of $\gamma \mid H$. Now since $H$ is normal every component of $\gamma \mid H$ is 1. Thus $\gamma \in H^{\perp}=A_{1}^{\prime}$ (cf. proof of Lemma 5). From Lemma 3(c) it follows that both $\alpha, \beta \in H^{\perp}$. Hence $\phi=1$. Thus $P \cap \bar{P}=\{1\}$ so that $P$ is an ordering semigroup for $\hat{H}$.

The following shows that $H$ is contained in the center of $G$.

Theorem 9. Let $H$ be a connected normal Abelian subgroup of $G$ and $P$ an ordering semigroup for $\hat{H}$. Suppose for each $\alpha \in \hat{G}$ the components of either $\alpha \mid H$ or $\bar{\alpha} \mid H$ all belong to $P$, then $H \subset$ center $G$.

In this case the components of $\alpha \mid H$ are all the same.

Proof. Suppose $s \in G$ and $G_{0}$ is the closed subgroup of $G$ generated by $H$ and $s$. The hypotheses of the theorem are satisfied if $G$ is replaced by $G_{0}$.

Let $a \in \hat{G}_{0}$ be such that the components of $\alpha \mid H$ belong to $P$. If $\alpha \notin H^{\perp}$ then

$$
\chi_{\alpha}(h)=\sum_{1}^{d(\alpha)} \phi_{i}(h) \quad(h \in H)
$$

where $\phi_{i} \in P$ and $\phi_{i} \neq 1$.

Now since $H$ is Abelian and connected the (matrices) $T_{\alpha}(h)$ can be simultaneously diagonalized for $h \in H$. It then follows that for $g \in G_{0}$

$$
\chi_{\alpha}(h g)=\sum_{1}^{d(\alpha)} a_{i}(g) \phi_{i}(h) \quad(h \in H) .
$$

Since $\phi_{i} \neq 1$ and $\chi_{\alpha}(h g)$ is analytic on $H$ we then have $\int_{H} \chi_{\alpha}^{n}(h g) d h=0$ 
$(n=1,2, \cdots)$. Hence, if $\alpha \in \hat{G}_{0}, \alpha \notin H^{\perp}$ (so that either $\alpha$ or $\bar{\alpha}$ is as above) and $d \tilde{g}$ is Haar measure on $G_{0} / H$ then for $n=1,2, \cdots$

$$
\int_{G_{0}} \chi_{\alpha}^{n}=\int_{G_{0} / H} d \tilde{g} \int_{H} \chi_{\alpha}^{n}(g h) d h=0 .
$$

Now suppose $G_{0}$ is non-Abelian so that there is $\beta \in \hat{G}_{0}$ with $\mathrm{d}(\beta)>1$. By the proof of Lemma 8 if $I=\alpha_{0}, \alpha_{1}, \cdots, \alpha_{n}$ are the distinct representations such that $\mu_{\beta, \bar{\beta}}\left(\alpha_{i}\right) \neq 0$ and $M_{i}=\left\{g \in G_{0}\right.$ : one is an eigenvalue of $\left.T_{\alpha_{i}}(g)\right\}$ then $\bigcup_{i=1}^{n} M_{i}=G_{0}$. Furthermore, if measure $M_{i}>0$ then for some positive integer $n_{i}$

$$
\int_{G_{0}} \chi_{\alpha_{i}}^{n_{i}} \neq 0
$$

By (1) it then follows that measure $M_{i}>0$ implies $\alpha_{i} \in H^{\perp}$.

Now $G_{0} / H$ is Abelian so that $\alpha_{i} \in H^{\perp}$ implies $d\left(\alpha_{\imath}\right)=1$. Thus there are representations $\alpha_{i}, \cdots, \alpha_{r} \in \hat{G}_{0}$ such that

(a) $\alpha_{i} \neq I, d\left(\alpha_{i}\right)=1$,

(b) $\alpha_{i} \in H^{\perp}$,

(c) measure $\cup_{i=1}^{r} M_{i}=1$.

Each $M_{i}$ is closed $(i=1, \ldots, r)$ since $d\left(\alpha_{i}\right)=1$ implies $M_{i}=\left\{g: \chi_{\alpha_{i}}(g)=1\right\}$. Hence by (c) $\bigcup_{i=1}^{r} M_{i}=G_{0}$. Thus $\chi_{\alpha_{i}}(s)=1$ for some $i$. But $\chi_{\alpha_{i}}(h)=1$ for $h \in H$ by (b). Hence $\chi_{\alpha_{i}} \equiv 1$ on $G_{0}$ which contradicts (a). Thus $G_{0}$ must be Abelian. That is, $s$ commutes with all elements of $H$. Hence $H C$ center $G$.

Proof of Theorem 2. Let $A$ be an $I$-Dirichlet algebra, $H$ the maximal antisymmetric subgroup and $P$ the ordering semigroup on $\hat{H}$ induced by $A$. To prove the first part of the theorem we need only show that $A \mid H$ consists of all functions which are analytic with respect to $P$. For, by the invariance of $A, A|H=A| H g$ and if $f \in C(G)$, $f|H g \in A| H g$ for all $g$ then, by Bishop's theorem [2], $\mathrm{f} \in A$.

If $\phi_{1} \in P$, there is $\chi_{\alpha} \in A$ such that $\chi_{\alpha} \mid H=n_{1} \phi_{1}+n_{2} \phi_{2}+\cdots$ where $\phi_{i}$ are distinct characters on $H$ and $n_{1} \neq 0$. Since $A \mid H$ is an $I$-algebra we have that $\phi_{1} \in A \mid H$. Thus $A \mid H$ contains all characters in $P$ and hence all functions which are analytic on $H$. Conversely each $f \in A$ is the uniform limit of finite sums of functions of the form $p(g)=\chi_{\alpha}\left(g_{0} g\right)$ where $\chi_{\alpha} \in A$. Each of these is analytic on $H$ and thus $f \mid H$ is analytic.

If $H$ is a closed, connected subgroup of center of $G$ and $P$ is an ordering semigroup for $\hat{H}$, let $\mathfrak{H}=\left\{\alpha \in \hat{G}:(d(\alpha))^{-1} \chi_{\alpha} \mid H \in P\right\}$. It is immediate that $\mathfrak{H C}$ is a subhypergroup, and $\mathcal{H}^{\prime}$ is an $I$-Dirichlet algebra.

If $K$ is the maximal antisymmetric subgroup for $\mathcal{H C}^{\prime}$ then 
$K^{\perp}=\left\{\alpha \in \hat{G}: \alpha, \bar{\alpha} \in\left(\mathcal{F C}^{\prime}\right)^{\prime}=\mathfrak{K}^{C}\right\}=H^{\perp}$. Thus $K=H$. Clearly the ordering semigroup of $\hat{H}$ induced by $\mathcal{H}^{\prime}$ is just $P$.

\section{REFERENCES}

1. Errett Bishop, $A$ generalization of the Stone-Weierstrass theorem, Pacific J. Math. 11 (1961), 777-783.

2. Irving Glicksberg, Measures orthogonal to algebras and sets of antisymmetry, Trans. Amer. Math. Soc. 105 (1962), 415-435.

3. Sigurdur Helgason, Lacunary Fourier series on noncommutative groups, Proc. Amer. Math. Soc. 9 (1958), 782-790.

4. Walter Rudin, Fourier analysis on groups, Interscience Tracts in Pure and Applied Mathematics, Interscience, New York, 1962.

Massachusetts Institute of TeChNology 